Artigo Original

\title{
Aprendizagem motora baseada em demonstrações de movimento biológico
}

\author{
Sérgio Tosi Rodrigues \\ Vânia Maria Castello \\ Juliana Gomes Jardim \\ Stefane Aline Aguiar
Laboratório de Informação, Visão e Ação (LIVIA), Departamento de Educação Física, Faculdade de Ciências, UNESP - Univ Estadual Paulista, Bauru, SP, Brasil

\begin{abstract}
Resumo: $O$ objetivo deste estudo foi avaliar o processo de aprendizagem motora de uma habilidade complexa da Ginástica Artística a partir da observação de demonstrações de modelos de pontos de luz e vídeo. Dezesseis participantes divididas em grupos dos respectivos modelos executaram um pré-teste, seguido de 100 tentativas de uma parada de mãos, igualmente distribuídas em blocos de 10 tentativas em dois dias, alternando períodos de demonstração e prática, com um teste de retenção após um dia. Cinemática de braço, tronco e perna das participantes possibilitaram análise da semelhança entre a coordenação de cada participante e do modelo e do tempo de movimento; a performance das participantes também foi avaliada por duas especialistas em Ginástica Artística. Ambas as análises indicaram que os grupos não diferiram. Os resultados são discutidos em termos da hipótese de suficiência de informação nos modelos de movimento biológico particularmente aplicada ao processo de aprendizagem de habilidades motoras complexas.
\end{abstract}

Palavras-chave: Percepção visual. Percepção de movimento. Desempenho psicomotor. Ginástica.

\section{Motor learning based on biological motion demonstrations}

\begin{abstract}
The aim of this study was to evaluate the motor learning process of a complex Artistic Gymnastics skill following demonstrations of point-light and video models. Sixteen participants divided into the respective model groups performed a pre-test and 100 trials of a handstand, equally distributed in blocks of 10 trials in two days, alternating periods of demonstration and practice, with retention test a day later. Participants' kinematics of arm, trunk, and leg was used to analyze the coordination similarities between each participant and the model and movement time; participants' performance was also evaluated by two Artistic Gymnastics experts. Both analyses revealed that the groups did not differ. Results are discussed in terms of the hypothesis of information sufficiency in biological motion models particularly applied to the learning process of complex motor skills.
\end{abstract}

Keywords: Visual perception. Motion perception. Psychomotor performance. Gymnastics.

\section{Introdução}

Professores de Educação Física e técnicos de modalidades esportivas fazem uso frequente de demonstração das habilidades motoras que estão ensinando. Através da observação visual (BLAKE; SHIFFRAR, 2007; BOUQUET et al., 2007), os aprendizes são capazes de perceber as ações de seus instrutores e produzi-las (HODGES et al., 2007).

Habilidades motoras complexas geram maior demanda cognitiva nos aprendizes porque envolvem maior número de elementos a serem controlados e maior grau de dificuldade na execução (NEWELL, 1985); os aprendizes necessitam de informação sobre os padrões cinemáticos que o organismo deve alcançar. Para estudar a percepção visual destes padrões, chamados de movimento biológico, Johansson (1973) criou uma técnica conhecida como animação de pontos de luz (BLAKE; SHIFFRAR, 2007), que preserva as propriedades cinemáticas do movimento humano e oferece informação útil nos ambientes de ensino (HORN; WILLIAMS; SCOTT, 2002). Imagens resultantes desta técnica contêm padrões de movimento dos pontos de luz executados por uma pessoa caminhando com pequenas luzes fixadas a sua cabeça e articulações em um ambiente completamente escuro (CUTTING; KOSLOWSKY, 1977). Desenvolvimentos mais recentes em ciência da computação e tecnologia têm permitido aos pesquisadores melhorarem a qualidade das 
animações de pontos de luz e manipular muitas variáveis de interesse (e.g., MA; PATERSON; POLLICK, 2006; VANRIE; VERFAILLIE, 2004).

Quando se assiste a uma animação de pontos de luz do caminhar, aspectos como a ação sendo executada (NORMAN et al., 2004), a identidade (FANI et al., 2005; TROJE; WESTHOFF; LAVROV, 2005) e o sexo de quem executa (TROJE, 2002) podem ser percebidos. É um tipo de percepção muito robusta mesmo quando os estímulos estão empobrecidos, ambíguos, ou são apresentados muito brevemente (BLAKE; SHIFFRAR, 2007).

O estudo de como a percepção deste movimento biológico modula a ação sendo aprendida pode ser feito com base na comparação de grupos que assistiram demonstrações de pontos de luz ou vídeo regulares (e.g., AL-ABOOD; DAVIDS; BENNETT, 2001; HAYES et al., 2007; HORN; WILLIAMS; SCOTT, 2002; HORN et al., 2005). A lógica subjacente ao teste é que se o movimento relativo entre as partes do corpo é informação suficiente para percepção e reprodução do movimento, as imagens de pontos de luz deveriam ser tão efetivas quanto às imagens de vídeo. Também tem sido mostrado que o uso de um modelo per se facilita o processo de aprendizagem motora, em comparação a grupos sem modelo ou métodos de orientação verbal ou descoberta $(\underline{\mathrm{AL}}-$ ABOOD; DAVIDS; BENNETT, 2001; HORN; WILLIAMS; SCOTT, 2002).

A remoção de informação estrutural através do uso de pontos de luz deveria facilitar a percepção de movimento em alguns casos porque evita informação não essencial, deixando saliente o movimento relativo entre as partes do corpo (RUNESON, 1984) e não fornecendo informação estrutural excessiva (NEWELL; WALTER, 1981). Terapeutas físicos foram capazes de fazer exames mais confiáveis da estabilização lombar a partir das imagens de pontos de luz do que das de vídeo (PELLECCHIA; GARRETT, 1997). No entanto, diferenças não foram observadas na percepção de qualidades estéticas e técnicas das rotinas de ginástica entre as fontes de vídeo e pontos de luz (SCULLY, 1986).

Os aprendizes adultos de uma sequência de ballet apresentaram uma posição de aterrissagem mais acurada e uma imitação mais próxima do padrão de movimento quando assistiram a um modelo de pontos de luz em comparação ao grupo que assistiu ao modelo de vídeo, tanto na velocidade regular quanto em câmera lenta (SCULLY; CARNEGIE, 1998). Na comparação do efeito de demonstrações de pontos de luz e vídeo sobre o processo de aprendizagem de um chute curto no futebol, diferenças nos resultados (medidas de erro radial e variável) e nos padrões de coordenação (amplitude articular de movimento e gráficos ângulo-ângulo) entre os grupos não foram encontradas; participantes que observaram o modelo (grupos de vídeo e pontos de luz) adquiriram um padrão de movimento global mais próximo ao movimento do modelo do que os controles (sem modelo), apesar deles não terem adquirido as relações locais no padrão de movimento, evidenciadas pelas medidas de coordenação (HORN; WILLIAMS; SCOTT, 2002). Resultados similares foram obtidos na comparação entre os grupos de vídeo, pontos de luz e sem-modelo na mesma tarefa no futebol. Os participantes que observaram os modelos mostraram mudanças imediatas e duradouras ao imitar mais fielmente o movimento relativo entre as partes do corpo do modelo. Eles também mediram o comportamento de busca visual (i.e., movimentos dos olhos) dos participantes, que foram mais seletivos no grupo de pontos de luz do que no grupo de vídeo, apesar da ausência de diferenças observada na acurácia da performance ou aprendizagem ( $\underline{\text { HORN }}$ et al., 2005).

De modo geral, a pesquisa revisada acima sugere que o conteúdo informacional dos modelos de pontos de luz é suficiente para o reconhecimento visual e a imitação do movimento apesar de algumas discrepâncias serem evidentes. Em particular, os resultados tendem a depender fortemente das demandas da tarefa; parece que quando a tarefa motora pode ser solucionada de muitos modos, não existe nenhuma vantagem em termos de resultado da performance em adotar a técnica demonstrada pelo modelo. De outro modo, quando não existe uma meta explícita da resposta, como na aprendizagem de uma habilidade da Ginástica Artística, a tarefa é menos restrita e a observação tem sua importância elevada ( 2007).

Apesar do debate sobre a saliência da informação através do uso de modelos de pontos de luz ter ganhado atenção na última década, talvez como um resultado dos avanços tecnológicos e conceituais (HODGES et al., 2007), estudos adicionais são necessários para esclarecer questões como as referentes à meta da resposta e à complexidade da tarefa. $O$ presente estudo combina características 
experimentais particulares, (i) com participantes executando uma tarefa altamente complexa, (ii) na qual não há uma meta explícita da resposta, (iii) com foco sobre a parte inicial da aprendizagem motora. Isto caracteriza uma contribuição específica à literatura. Estas características foram planejadas para maximizar os efeitos das apresentações de pontos de luz, conforme sugerido por Rodrigues, Ferracioli e Denardi (2010) em estudo da pirouette do ballet. Estes autores observaram a referida suficiência, mas não a maior relevância em comparação à informação de vídeo comum. Diferenças entre características das ações complexas sob investigação, tais como eixos e planos principais do movimento e a base de apoio nos pés ou mãos, tornam distintos a informação visual fornecida nos modelos de vídeo e os requerimentos de execução dos aprendizes; estas diferenças parecem ter potencial de alterar os indicativos referentes à suficiência e relevância dos modelos. Neste sentido, o presente estudo formulou questões semelhantes às de Rodrigues, Ferracioli e Denardi (2010), mas utilizou, exploratoriamente, uma habilidade complexa distinta, a parada de mãos.

O objetivo do presente estudo foi comparar o processo de aprendizagem de uma habilidade complexa da Ginástica Artística baseada em demonstrações de modelos de pontos de luz e vídeo. Estabeleceu-se a hipótese que 0 movimento relativo entre as partes do corpo, saliente no modelo de pontos de luz, oferece informação suficiente e mais relevante para o reconhecimento e reprodução do movimento do que o modelo de vídeo, favorecendo o processo de aprendizagem da parada de mãos. Para testar esta hipótese, a coordenação do corpo todo foi analisada em termos de escores de performance global atribuídos por profissionais de Ginástica Artística. Especificamente, diferenças na coordenação ombro-quadril em relação ao modelo e tempo de movimento na tarefa foram usadas para descrever a performance. Esperouse que o grupo de pontos de luz apresentasse valores significativamente melhores em todas variáveis medidas, indicando o uso de informação saliente disponível no modelo de pontos de luz durante o processo de aprendizagem motora.

\section{Método}

\section{Participantes}

Dezesseis estudantes do nível de graduação, do sexo feminino, com idade média de 20,7 anos $(\mathrm{DP}=1,92)$ e acuidade visual normal ou corrigida para normal participaram como voluntárias do presente estudo. As participantes, sem nenhuma experiência em Ginástica Artística e não familiares com os propósitos do estudo, foram aleatoriamente divididas nos grupos de vídeo completo (VID) e pontos de luz (PL), resultando em dois grupos de oito participantes. Antes do experimento, as participantes leram e assinaram um termo de consentimento descrevendo os procedimentos experimentais aos quais foram submetidos, conforme as exigências do comitê local de ética em pesquisa.

\section{Equipamentos}

Uma câmera de vídeo (Sony DCR-DVD205), com frequência de gravação padrão $(60 \mathrm{~Hz})$, formato digital DVD, foi utilizada neste estudo. Os softwares Studio 10.5 (Pinnacle Systems) e Ariel Performance Analysis System (APAS) - versão 1.0 - foram utilizados para edição e análise cinemática. O software Flash Mx (Macromedia) gerou as imagens de PL. Uma televisão (Sony K29T76, $22 \times 16$ polegadas) foi utilizada para apresentação das demonstrações às participantes. Os softwares Matlab (Mathworks, version5) e SPSS (SPSS, version 9) foram utilizados para o cálculo de variáveis e análises estatísticas.

\section{Tarefa e construção dos filmes de teste}

A tarefa foi uma parada de mãos, que é uma habilidade complexa da Ginástica Artística; ela envolve a manutenção do equilíbrio corporal no apoio invertido (i.e., com as mãos em contato com o solo). O executante deve manter punhos, cotovelos, ombros, quadris, joelhos e tornozelos alinhados à vertical, como mostra a Figura 1. Os requerimentos combinados de controle do equilíbrio com área reduzida de contato com o solo, força muscular para estabilizar a postura e suavidade do movimento para preservar a qualidade artística podem demonstrar o seu nível de dificuldade. Esta tarefa foi escolhida pelo número apropriado de detalhes e facilidade de adequação às condições experimentais; esperouse que demonstrações fossem mais efetivas durante a fase inicial do processo de aprendizagem, que está sob investigação no presente estudo. A meta principal para ambos os grupos era imitar o padrão de movimento da modelo apresentado nas imagens de uma tela de televisão: o grupo VID assistiu a performance da modelo em formato de vídeo regular (Figura 1A) enquanto 0 grupo $\mathrm{PL}$ assistiu a uma versão editada do mesmo vídeo mostrando apenas os pontos de luz sobre um fundo preto (Figura 1B). 


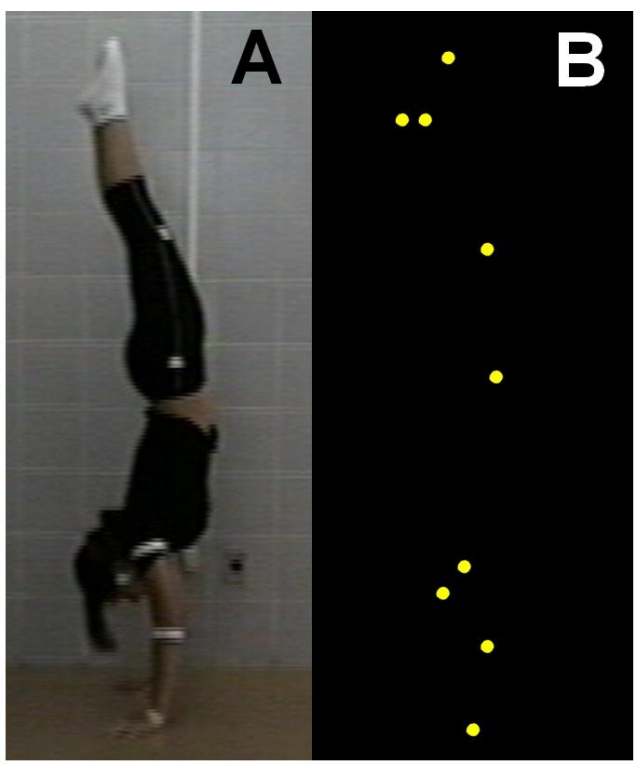

Figura 1. Quadros ilustrativos da execução da parada de mãos pelo modelo nos formatos das imagens de vídeo (A) e pontos de luz (B).

As características do vídeo de PL foram definidas através de um teste prévio de reconhecimento do movimento biológico; o vídeo continha 13 marcadores amarelos localizados na orelha, ombro, cotovelo, punho, quadril (do lado esquerdo), joelho, tornozelo, calcanhar e ponta do pé (em ambos os lados). Trinta estudantes do nível de graduação de ambos os sexos, com idade média de 20,5 anos $(D P=1,87)$, com acuidade visual normal ou corrigida para normal, participaram deste teste. O vídeo com o nível de reconhecimento mais alto $(93,3 \%$ reconheceram a habilidade na primeira apresentação e 0 restante precisou assistir duas ou três vezes; $70,0 \%$ reportaram verbalmente serem capazes de executar a habilidade apenas com a informação visualizada) foi utilizado no presente experimento.

\section{Procedimentos e delineamento}

Antes da coleta de dados, as participantes de ambos os grupos receberam instruções para assistirem atentamente a modelo durante a fase de observação e, então, copiarem exatamente seus movimentos durante a fase de prática. Marcadores de isopor (diâmetro de $3 \mathrm{~cm}$ ) foram afixados no punho (processo estilóide da ulna), ombro (região da cabeça e tuberosidade do úmero), quadril ( $3 \mathrm{~cm}$ acima do trocanter maior do fêmur) e tornozelo (extremidade distal do maléolo lateral da fíbula) (ZATSIORSKY, 1998). Participantes de ambos os grupos tiveram um pré-teste, seguido de um total de 100 tentativas de prática igualmente divididas em blocos de 10 tentativas durante dois dias consecutivos de coleta de dados. Antes do pré-teste e de cada bloco, os participantes assistiram três vezes ao seu respectivo modelo de vídeo (VID ou PL), sentados a 2,4 $\mathrm{m}$ da tela da televisão. $O$ ângulo visual da imagem da modelo na retina das participantes foi aproximadamente 8,6 graus no plano horizontal e 8,3 graus no plano vertical. Durante a prática, conhecimento dos resultados / performance não foi oferecido após a execução, porque isto poderia afetar os grupos diferentemente ( $\underline{\text { HORN }}$ et al., 2005). Intervalos de dois minutos entre os blocos e a qualquer momento sob solicitação da participante foram usados para descanso. Após um dia, as participantes foram submetidas a um teste de retenção executando um bloco de prática final. As participantes tiveram, além do pré-teste, a décima tentativa de cada bloco de prática e a décima tentativa do bloco de retenção filmadas na perspectiva lateral para análise posterior.

\section{Variáveis dependentes e análise dos dados}

Escore de performance global. Duas especialistas em Ginástica Artística, após assistirem o vídeo de cada tentativa gravada, atribuíram notas (de zero a dez) a cada execução da parada de mãos de acordo com a proximidade técnica entre a performance observada e a performance da modelo; os respectivos valores médios foram usados para quantificarem a performance global para cada indivíduo. Um escore dez representou um movimento idêntico à demonstração da modelo. Cada especialista avaliou um conjunto de dados randomicamente ordenados de todas tentativas de todos participantes e um conjunto adicional de $20 \%$ das tentativas para cada participante. Assim, durante a avaliação de cada tentativa, as especialistas não estavam cientes do grupo dos participantes ou do número da tentativa, ou sobre qual participante estaria na próxima tentativa a ser vista. A correlação de Pearson entre as avaliações das duas especialistas foi de $0,84(p<$ $0,001)$. Para os $20 \%$ das tentativas avaliadas duas vezes por cada especialista, as correlações entre a primeira e a segunda avaliação foram de 0,91 e 0,90 ( $p s<0,001$ ), respectivamente para as especialistas 1 e 2 .

Diferença normalizada do quadrado da raiz média (NoRM-D). A análise cinemática foi realizada nos dados da modelo e dos participantes; a relação entre ombro (braço e tronco) e quadril (tronco e perna) durante a ação da parada de mãos serviu como um critério para comparar os grupos. Mudanças na coordenação ombro-quadril durante as tentativas de prática e 
retenção foram usadas para acessar os efeitos de assistir imagens de modelos de VID ou PL. Os pontos de início e término das tentativas foram, respectivamente, os instantes de contato inicial da mão com o solo e de contato final do pé com o solo em sua fase descendente, após o período de equilíbrio apoiado nas duas mãos. Os dados foram filtrados com um filtro Butterworth de quarta ordem e frequência de corte de $2 \mathrm{~Hz}$; a frequência de corte foi definida através de análise residual (WINTER, 1990). Uma interpolação do tipo "spline" foi executada para normalizar este período a 101 pontos de dados, possibilitando a comparação com a modelo ao longo das tentativas.

A proximidade entre o padrão de coordenação ombro-quadril no espaço das articulações entre participante e modelo foi medido pela diferença normalizada do quadrado da raiz média (NoRMD), como usado em estudos anteriores (HAYES et al., 2007; HORN et al., 2005). Este escore foi uma versão modificada do erro normalizado do quadrado da raiz média (SIDAWAY; HEISE; SCHOENFELDER-ZHODI, 1995), que usa a disparidade de cada tentativa com os dados da modelo em cada tentativa; para detalhes do cálculo, veja Mullineaux, Bartlett e Bennett (2001).

Tempo de movimento (TM). Tempo passado entre o início e o término da execução da parada de mãos; equivale à duração total da tentativa.

\section{Análise estatística}

Dados de cada variável dependente obtida durante a fase de prática foram submetidos a uma análise de variância de Grupo (VID, PL) por Tentativas (Pré, 100), com medidas repetidas no último fator. Dados de todas variáveis dependentes obtidas no final da fase de prática e após um dia foram submetidos a uma análise de variância de Grupo (VID, PL) por Tentativas (100, Retenção), com medidas repetidas no último fator. Nas duas análises de variância, a variável escore de performance global contou adicionalmente com o efeito principal Especialista (1 e 2). Quando violações do pressuposto de esfericidade para análise de variância com medidas repetidas foram observadas, ajustes de Greenhouse-Geisser foram usados; comparações aos pares através do teste de Tukey e ajustes de Bonferroni para múltiplas comparações foram utilizados conforme necessário (MAXWELL; DELANEY, 1990). O nível de significância adotado foi 0,05 para todas as análises.

\section{Resultados}

Os escores de performance global tiveram um aumento significativo durante a fase de aquisição, com valores da última tentativa da fase de aquisição $(M=6,56, E P=0,53)$ sendo superiores aos do Pré-teste $(M=5,29, E P=0,46), F(1,14)=$ $12,81, p=0,003$ (Figura 2). Os escores obtidos pelos grupos VID e PL foram semelhantes; os efeitos de Grupo assim como da interação Grupo por Tentativas não foram significativos sobre esta variável. A análise de variância também revelou diferença significativa entre os escores das duas especialistas, $F(1,14)=8,61, p=0,011$. O valor médio dos escores de performance global atribuídos pela especialista 1 foi $5,61(E P=0,48)$ e pela especialista 2 foi $6,23 \quad(E P=0,47)$. Interessantemente, os escores de performance global obtiveram um aumento significativo entre a última tentativa da fase de aquisição $(M=6,19$, $E P=0,55)$ e o teste de retenção $(M=6,97, E P=$ $0,50), F(1,14)=9,24, p=0,009$; não houve efeito significativo da especialista, de Grupo nem da interação Grupo por Tentativa no teste de retenção.

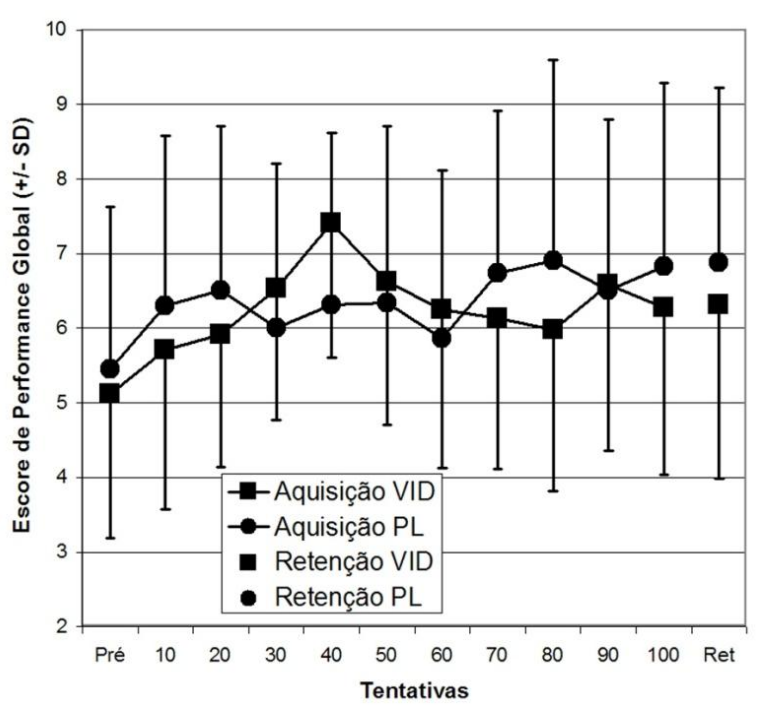

Figura 2. Escore de performance global em função das tentativas de aquisição e retenção para os grupos de vídeo (VID) e pontos de luz $(\mathrm{PL})$.

$\mathrm{Na}$ fase de aquisição, os valores de NoRM-D não foram significativamente afetados por Grupo, Tentativa ou interação Grupo por Tentativa. A Figura 3 mostra uma ligeira redução nos valores de NoRM-D ao longo das tentativas, mas a diferença entre o pré-teste $(M=45,62, E P=2,50)$ e a última tentativa desta fase $(M=40,21, E P=$ $3,47)$ não foi suficiente para alcançar significância, $F(1,14)=3,03, p=0,103$. No teste 
de retenção, de modo similar, os valores de NoRM-D não foram significativamente afetados por Grupo, Tentativa ou interação Grupo por Tentativa. No entanto, os ângulos do ombro e do quadril usados para calcular NoRM-D mostraram que, tanto para VID quanto para PL, o padrão de movimento do ombro (Figuras 4A e 4C) parece alcançar estabilidade semelhante à da modelo por maior período durante cada tentativa do que o observado no movimento do quadril (Figura 4B e 4D). A referida estabilidade reduzida equivale à manutenção da extensão da articulação do quadril durante a fase mais crítica da posição invertida da parada de mãos. Vale salientar que as curvas dos ângulos do ombro e do quadril mostrados na Figura 4 representam dados separados por grupo durante todas tentativas, uma vez que não foram observados efeitos significativos de tentativa para a variável NoRM-D nas fases de aquisição e retenção do experimento.
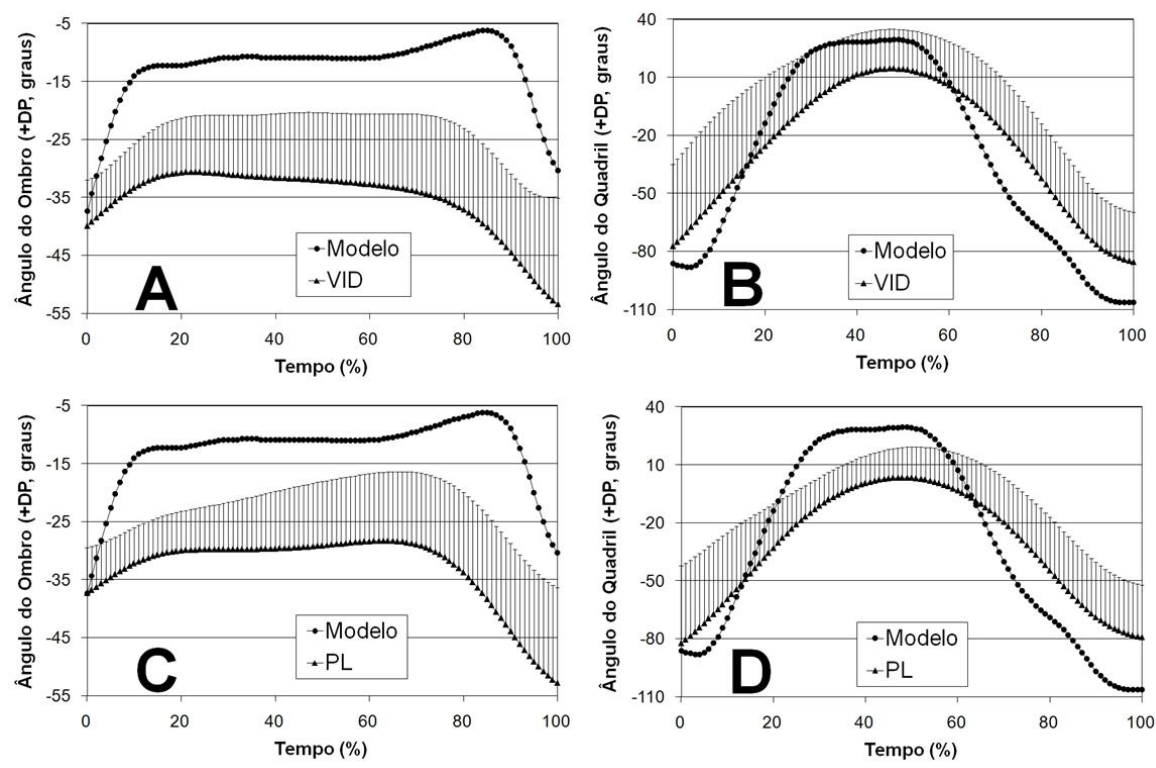

Figura 4. Ângulo (graus) do ombro ( $A$ e $C$ ) e do quadril (B e D) da modelo e das participantes de VID e PL, respectivamente, em função do tempo relativo (\%) da tentativa. As barras de erro indicam um desvio padrão.

Houve uma pequena elevação dos valores do TM ao longo das tentativas, similar para as participantes do grupo VID e PL; o aumento de TM do pré-teste $(M=2,36 \mathrm{~s}, E P=0,12)$ até a última tentativa da fase de aquisição $(M=2,62 \mathrm{~s}$, $E P=0,17)$ não alcançou significância. Nesta fase de aquisição, os valores de TM foram equivalentes para ambos os grupos, em média 2,49 s $(E P=0,13)$; a interação Grupo por Tentativa também não afetou significativamente os valores de TM. Na fase de retenção, de modo semelhante, o TM não foi significativamente

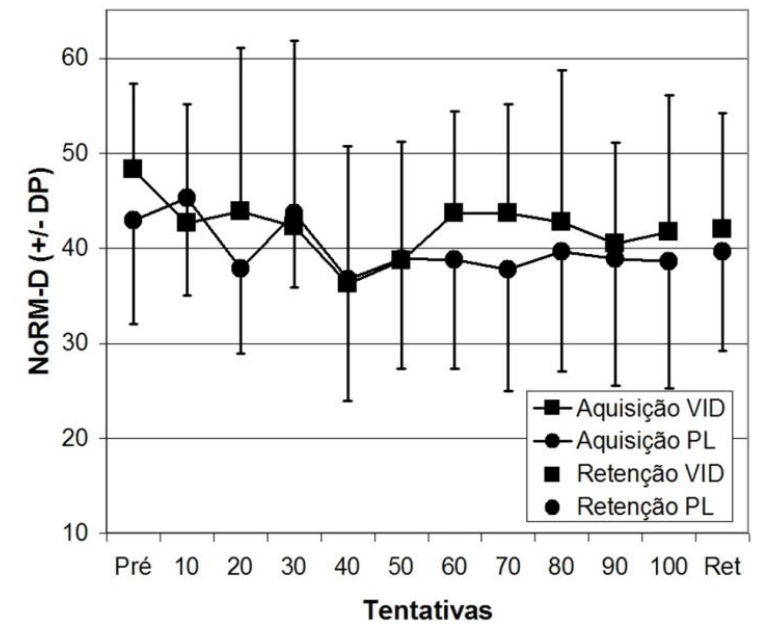

Figura 3. Diferença normalizada do quadrado da raiz média (NoRM-D) em função das tentativas de aquisição e retenção para os grupos de vídeo (VID) e pontos de luz (PL).O TM da parada de mãos não aumentou significativamente durante a fase de aquisição, $F(1,14)=2,68, p=0,12$. afetado pelos efeitos de Grupo e Tentativa, nem pela interação Grupo por Tentativa.

\section{Discussão}

A hipótese testada neste estudo foi a de que um processo de aprendizagem motora mais efetivo da parada de mãos ocorreria para o grupo PL porque os modelos de movimento biológico presumivelmente preservam e salientam a informação visual necessária. Esperava-se que os resultados do grupo $\mathrm{PL}$ fossem superiores aos do grupo VID, decorrentes da prática ocorrida durante $o$ experimento. 
Os resultados presentes não confirmaram a esperada superioridade dos resultados do grupo PL no processo de aprendizagem. Os valores do Escore de Performance Global (Figura 2), da NoRM-D (Figura 3) e do TM mostraram comportamento similar entre os grupos VID e PL nas fases de aquisição e retenção, corroborando a noção que a informação disponibilizada pelos modelos de movimento biológico é suficiente para o reconhecimento do movimento e sua reprodução, mas não é mais relevante do que no vídeo regular. A literatura sobre aprendizagem motora tem mostrado ausência similar de efeitos relacionados ao tipo de modelo de vídeo. Estudos anteriores sobre aprendizagem motora baseada em modelos de movimento biológico (HORN; WILLIAMS; SCOTT, 2002; HORN et al., 2005) não observaram diferenças nas medidas motoras entre os grupos VID e PL durante a aprendizagem de um chute curto do futebol de campo; apenas um aspecto perceptual, o padrão de busca visual, mostrou-se mais seletivo para o grupo PL do que para o grupo VID (HORN et al., 2005). Semelhança nas variáveis motoras entre os grupos VID e PL também foi observada durante a aprendizagem da pirouette do ballet (RODRIGUES; FERRACIOLI; DENARDI, 2010). Durante o processo de aprendizagem de um lançamento do boliche, grupos adultos de PL e VID não revelaram diferenças na forma do movimento, apesar das crianças terem uma performance mais pobre quando observando 0 modelo de PL em comparação ao modelo de VID (HAYES et al., 2007); esta inferioridade das crianças que observaram $\mathrm{PL}$ não pode ser explicada pela diferença no desenvolvimento, uma vez que bebês com um ano de idade já são capazes de perceber movimento biológico (BERTENTHAL; PROFFITT; CUTTING, 1984). Contudo, os resultados do presente estudo, obtidos com adultos jovens, parecem sugerir que o alto nível de complexidade da tarefa e a ausência de uma meta explícita da resposta, características da parada de mãos que supostamente valorizariam a demonstração e potencializariam diferenças entre os grupos, não foram capazes de alterar o uso da informação visual contida no modelo de movimento biológico para favorecer uma melhor aprendizagem motora.

Uma melhoria perceptual-motora em decorrência da prática era esperada para ambos os grupos. Os resultados presentes dão suporte parcial a esta expectativa. Apesar dos valores de NoRM-D (Figura 3) e MT mostrarem alguma tendência de melhoria ao longo da prática, principalmente nas 30-40 tentativas iniciais, estas mudanças não atingiram significância até o final da fase de aquisição, nem foram confirmadas no teste de retenção. De outro modo, os Escores de Performance Global atribuídos pelas especialistas em Ginástica Artística indicaram uma melhoria significativa na semelhança entre a performance de cada grupo e a performance do modelo, quando comparados o pré-teste e o último bloco da fase de aquisição, após 100 tentativas de prática. Interessantemente, a comparação entre este último bloco e o de retenção mostrou nova melhoria significativa nos Escores de Performance Global, indicando que a performance de ambos os grupos ainda não havia estabilizado quando o experimento foi encerrado. Assim, os presentes resultados mostram discrepâncias na caracterização do processo de aprendizagem motora das participantes ao longo da fase de aquisição, se comparadas as variáveis Escore de Performance Global, NoRM-D e TM.

Estas discrepâncias poderiam ser atribuídas ao efeito da quantidade necessária de prática distinta para a melhoria nos elementos espaciais e temporais do movimento; as características de movimento altamente habilidoso da modelo, contidas nos vídeos, não foram necessariamente adquiridas simultaneamente durante seu longo período de prática (RODRIGUES; FERRACIOLI; DENARDI, 2010). No caso da habilidade pirouette do ballet, como bailarinas experientes parecem não mudar seus TM mesmo executando com os olhos vendados - o que indica que a escala de tempo da rotação do tronco não é dependente de atualizações sensoriais (DENARDI; FERRACIOLI; RODRIGUES, 2008) - a observação do TM sendo consistentemente reduzido ao longo da prática e dos valores de NoRM-D inalterados ao longo de 160 tentativas de prática foi interpretada como indicativo que a quantidade de prática do experimento foi suficiente para promover melhorias relevantes apenas na estrutura temporal do movimento do aprendiz e que aspectos espaciais como a similaridade com a dinâmica de coordenação do modelo (NoRM-D) e - controle postural (oscilação corporal) possivelmente requerem uma maior quantidade de prática do que a utilizada para promover melhoria na performance (RODRIGUES, 2006). De modo semelhante, tem sido mostrado que os benefícios de observar o modelo, comparado ao grupo controle (sem demonstração), foram limitados aos fatores temporais; os participantes que observaram o modelo de vídeo apresentaram uma clara redução na variabilidade de fase temporal ao longo das tentativas de prática enquanto os outros grupos apresentaram uma variabilidade equívoca (HORN; WILLIAMS; 
SCOTT, 2002). No presente estudo, o TM não foi significativamente alterado ao longo da prática evidenciando uma aprendizagem mais rápida de elementos temporais, mas os dados parecem sugerir que a similaridade dos ângulos do quadril e ombro entre aprendiz e modelo, uma característica espacial, requer uma quantidade de prática superior às 100 tentativas utilizadas, suficiente para gerar redução significativa em seus valores. Alternativamente, vale reconhecer que a variável TM incluía a duração total do movimento da parada de mãos enquanto 0 aspecto mais saliente de diferença temporal entre o modelo e cada aprendiz foi o período de estabilidade do ângulo do quadril (Figuras 4B e 4D), que representou somente $20 \%$ da duração total, possivelmente um período muito curto para reduzir suficientemente a variável TM ao longo da prática. Por outro lado, os Escores de Performance Global, que obtiveram melhoria significativa durante as fases de aquisição e retenção, parecem representar aspectos diferentes do movimento de braços, tronco e pernas, com maiores detalhes e sutilezas não contidos na medida NoRM-D. A avaliação mais subjetiva das especialistas em Ginástica Artística, que envolve aspectos tanto espaciais quanto temporais, foi capaz de determinar a melhoria esperada na performance das aprendizes com base nestas informações adicionais. Cabe enfatizar que apesar de diferença significativa no rigor da avaliação da fase de aquisição, com a Especialista 1 sendo mais conservadora $(M=5,6)$ do que a Especialista $2(M=6,2)$, a consistência entre elas e para cada uma delas quando avaliou duas vezes a mesma tentativa (correlações de $0,84,0,90$ e 0,91 , respectivamente) foi bastante elevada.

Um aspecto adicional da percepção de movimento biológico pode estar relacionado à ausência de efeitos da prática nos valores de NoRM-D. Os grupos VID e PL não se tornaram mais semelhantes ao padrão de movimento do modelo possivelmente porque 0 controle do ângulo do quadril, com os joelhos estendidos na posição inversa, e a manutenção desta posição por longos períodos é um aspecto técnico da parada de mãos observado apenas em ginastas muito experientes. $O$ modelo poderia inclusive permanecer na parada de mãos por período muito mais longo do que o utilizado na confecção do vídeo de demonstração. Para ambos os grupos, o período de manutenção dos valores do ângulo do ombro mais elevados durante o tempo relativo da parada de mãos foi muito mais próximo ao do modelo (Figura 4A e 4C) do que este período para o ângulo do quadril (Figura 4B e 4D); as participantes de VID e PL não foram capazes de manter a estabilidade na posição inversa por algum tempo, alçando gradativamente o ápice por volta dos $50 \%$ do tempo e retornando à posição inicial imediatamente. Diferentemente, o modelo alcançou mais rapidamente o ápice, por volta dos $30 \%$, e manteve o equilíbrio postural até os $50 \%$, retornando também mais abruptamente à posição inicial. Diferentes formas de coordenação entre partes do corpo foram observadas em habilidade do ballet. Bailarinas com experiência média de 12 anos de prática usaram uma estratégia particular para manter a estabilidade visual: durante a pirouette, o tronco inicia a rotação primeiro, seguida sequencialmente pela cabeça e pelo olhar; no final, o olhar chega primeiro no mesmo ponto de fixação inicial a frente, seguido pela cabeça e, finalmente, tronco (DENARDI; FERRACIOLI; RODRIGUES, 2008). Interessantemente, quando estas bailarinas experientes estavam vendadas as variáveis afetadas significativamente foram a duração da rotação da cabeça, a diferença entre as rotações tronco-cabeça e as oscilações anteroposteriores da cabeça e do tronco indicando que estas variáveis estão associadas com os elementos que são mais difíceis e requerem mais prática (DENARDI; FERRACIOLI; RODRIGUES, 2008). No presente estudo, havia informação disponível específica nos dois tipos de modelo de vídeo sobre o período de manutenção ao longo da tentativa de valores máximos dos ângulos do quadril e do cotovelo, o que potencialmente poderia ser usado pelas aprendizes para aumentar as referidas estabilizações ao longo da prática. De outra forma, os dois grupos foram capazes de executar de modo mais semelhante ao modelo, com mais longo período de estabilidade de valores máximos, na posição inversa, somente para articulação do ombro; nenhum dos grupos foi capaz de se aproveitar da disponibilidade de informação para reproduzir na ação motora o período de estabilidade de valores máximos da articulação do quadril - este achado foi interpretado como insuficiência da quantidade de prática durante 0 experimento. De modo alternativo, como mencionado acima, o problema poderia ter sido perceptual, com dificuldade das participantes em identificar no vídeo um período de estabilidade relativamente curto ( 20\%), referente ao quadril, no contexto do movimento completo, o que parece ser a informação mais relevante para este aspecto da tarefa.

Estudos anteriores têm sugerido que tarefas muito simples (HORN; WILLIAMS; SCOTT, 2002), requerimentos de meta da resposta (HODGES et al., 2007) e estágio mais avançado de aprendizagem (NEWELL, 1985) poderiam mascarar diferenças entre os grupos PL e VID. 
Para superar estas limitações, o presente estudo usou uma tarefa altamente complexa, que não possuía uma meta explícita da resposta, durante a fase inicial da aprendizagem motora. As evidências presentes dão suporte à hipótese de suficiência da informação para aprendizagem motora em modelos de movimento biológico. Especificamente, 0 uso de uma habilidade altamente complexa trouxe outros aspectos ao debate PL-VID, como as diferenças entre 0 controle das articulações do ombro e do quadril durante a aprendizagem de um padrão de coordenação complexo. Novos estudos parecem necessários para isolar de modo sistemático os efeitos dos modelos de movimento biológico durante a aprendizagem de habilidades motoras complexas e esclarecer se há uma forte dependência dos resultados com relação às restrições da tarefa observadas até o momento.

\section{Referências}

AL-ABOOD, S. A.; DAVIDS, K.; BENNETT, S. J. Specificity of task constraints and effects of visual demonstrations and verbal instructions in directing learners search during skill acquisition. Journal of Motor Behavior, Washington, v. 33, p. 295-305, 2001.

BERTENTHAL, B.; PROFFITT, D.; CUTTING, J. Infant sensitivity to figural coherence in biomechanical motion. Journal of Experimental Child Psychology, New York, v. 37, p. 213-230, 1984.

BLAKE, R.; SHIFFRAR, M. Perception of human motion. Annual Review of Psychology, Palo Alto, v. 58, p. 47-73, 2007.

BOUQUET, C. A.; GAURIER, T.; SHIPLEY, T.; TOUSSAINT, L.; BLANDIN, Y. Influence of the perception of biological or non-biological motion on movement execution. Journal of Sports Sciences, London, v. 25, p. 519-530, 2007.

CUTTING, J. E.; KOSLOWSKI, L. Recognising friends by their walk: Gait perception without familiary cues. Bulletin of Psychonomic Society, Austin, v. 9, p. 353-356, 1977.

DENARDI, R. A.; FERRACIOLI, M. C.; RODRIGUES, S. T.Informação visual e controlo postural durante a execução da pirouette no ballet. Revista Portuguesa de Ciência do Desporto, Porto, v. 8, n. 2, p. 241-250, 2008.

FANI, L.; PRASAD, S.; HARBER, K.; SHIFFRAR, $M$. Recognizing people from their movements. Journal of experimental psychology: Human perception and performance, Washington, v. 31, p. 210-220, 2005.
HAYES, S. J.; HODGES, N. J.; SCOTT, M. A.; HORN, R. R.; WILLIAMS, A. M. The efficacy of demonstrations in teaching children an unfamiliar movement skill: The effects of object-orientated actions and point-light demonstrations. Journal of Sports Sciences, London, v. 25, p. 559-575, 2007.

HODGES, N. J.; WILLIAMS, A. M.; HAYES, S. J.; BRESLIN, G. What is modelled during observational learning? Journal of Sports Sciences, London, v. 25, p. 531-545, 2007.

HORN, R. P.; WILLIAMS, A. M.; SCOTT, A. M. Learning from demonstration: The role of visual search during observational learning from video and point-light models. Journal of Sports Sciences, London, v. 20, p. 253-269, 2002.

HORN, R. R.; WILLIAMS, M. A.; SCOTT, M. A.; HODGES, N. J. Visual Search and coordination changes in response to video and point-light demonstrations without KR. Journal of Motor Behavior, Washington, v. 37, p. 265-274, 2005.

JOHANSSON, G. Visual perception of biological motion and a model for its analysis. Perception \& Psychophys, Austin, v. 14, p. 201-211, 1973.

MA, Y.; PATERSON, H. M.; POLLICK, F. E. A motion capture library for the study of identity, gender and emotion perception from biological motion. Behavior Research Methods, Austin, v. 38, p. 134-141, 2006.

MAXWELL, S. E.; DELANEY, H. D. Designing experiments and analyzing data: A model comparison perspective. Pacifc Grove, CA: Brooks/Cole, 1990, 902 p.

MULLINEAUX, D. R.; BARTLETT, R. M.; BENNETT, S. Research design and statistics in biomechanics and motor control. Journal of Sports Sciences, London, v. 19, p .739-760, 2001.

NEWELL, K. M. Coordination, control and skill. In: GOODMAN, D.; WILBERG, R. B.; FRANKS, I. M. (Eds.). Differing perspectives in motor learning, memory and control. Amsterdam: Elsevier, 1985, p. 295-317.

NEWELL, K. M.; WALTER, C. B. Kinematic and kinetic parameters as information feedback in motor skill acquisition. Journal of Human Movement Studies, New York, v. 7, p. 235-254, 1981.

NORMAN, J. F.; PAYTON, S. M.; LONG, J. R.; HAWKES, L. M. Aging and the perception of biological motion. Psychology and Aging, Arlington, v. 19, p. 219-225, 2004. 
PELLECCHIA, G. L.; GARRETT, G. E. Assessing lumbar stabilization from point-light and normal video displays of manual lifting. Perceptual \& Motor Skills, Missoula, v. 85, p. 931-937, 1997.

RODRIGUES, S. T. O mundo visual da bailarina: Percepção-ação durante a pirouette. Revista Brasileira de Educação Física e Esporte, São Paulo, v. 20, p. 103-106, 2006.

RODRIGUES, S. T.; FERRACIOLI, M. C.; DENARDI, R. A. Learning a complex motor skill from video and point-light demonstrations.

Perceptual \& Motor Skills, Missoula, v. 111, p. 307-323, 2010.

RUNESON, S. Perception of biological motion: The KSD principle and the implications of a distal versus proximal approach. In: JANSSON, G.; BERGSTOM, S.; EPSTEIN, W. (Eds.).

Perceiving events and objects. Hillsdale, $\mathrm{NJ}$ : Erlbaum, 1984, p. 383-405.

SCULLY, D.; CARNEGIE, E. Observational learning in motor skill acquisition. Irish Journal of Psychology, Dublin, v. 19, p. 472-485, 1998.

SCULLY, D. M. Visual perception of aesthetic quality and technical execution in biological motion. Human Movement Science, Amsterdam, v. 5, p. 185-206, 1986.

SIDAWAY, B.; HEISE, G.; SCHOENFELDERZHODI, B. Quantifying the variability of angle angle plots. Journal of Human Movement Studies, New York, v. 29, p. 181-197, 1995.

TROJE, N. F. Decomposing biological motion: A framework for analysis and synthesis of human gait patterns. Journal of Visualized

Experiments, Cambridge, v. 2, p. 371-387, 2002.

TROJE, N. F.; WESTHOFF, C.; LAVROV, M. Person identification from biological motion: effects of structural and kinematic cues.

Perception \& Psychophysics, Austin, v. 67, p. 667-675, 2005.

VANRIE, J.; VERFAILLIE, K. Perception of biological motion: A stimulus set of human pointlight actions. Behavioral Research Methods, New York, v. 36, p. 625-629, 2004.

WINTER, D. A. Biomechanics and Motor Control of Human Movement. New York: Wiley, 1990, $277 \mathrm{p}$.

\section{ZATSIORSKY, V. M. Kinematics of human}

motion. Champaign, IL: Human Kinetics, 1998, $419 \mathrm{p}$.
Agradecimentos: Este estudo obteve apoio através de bolsas de estudo a Vânia Maria Castello, Juliana Gomes Jardim (ambas do $\mathrm{PIBIC/CNPq)} \mathrm{e} \mathrm{Stefane} \mathrm{Aline} \mathrm{Aguiar} \mathrm{(FAPESP,}$ processo 2009/00899-8). Os autores agradecem M.Sc. Rodrigo Carvalho (CTI "Isaac Portal Roldán", UNESP/Bauru) pelo apoio técnico na construção dos modelos de vídeo.

\section{Endereço:}

Sérgio Tosi Rodrigues Unesp - Departamento de Educação Física. Av. Luis Edmundo Carrijo Coube, 14-01 Vargem Limpa

Bauru SP Brasil 17033-360

Telefone: (14) 3103-6082

e-mail: srodrigues@fc.unesp.br

Recebido em: 9 de agosto de 2011. Aceito em: 23 de setembro de 2012.

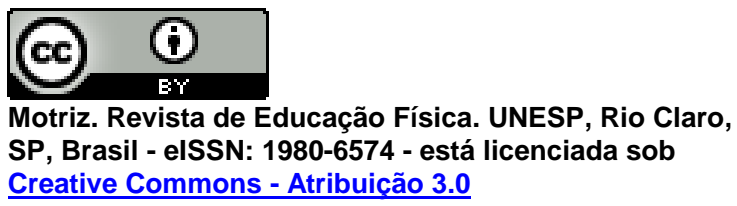
SP, Brasil - elSSN: 1980-6574 - está licenciada sob Creative Commons - Atribuicão 3.0 